\title{
The temporal pattern of VR sickness during 7.5-h virtual immersion
}

\author{
Shanshan Chen ${ }^{1} \cdot$ Dongdong Weng ${ }^{1,2}$
}

Received: 1 March 2021 / Accepted: 30 September 2021 / Published online: 30 October 2021

(c) The Author(s) 2021

\begin{abstract}
In this study, we assessed the relationship between exposure duration and VR sickness severity during 7.5-h virtual immersion. First, we showed that the VR sickness severity was positively correlated to the exposure duration: the longer participants were exposed to the VR environment, the more severe sickness symptoms they had. Second, we showed a dynamic sickness adaptation process during a long time of VR exposure: the sickness adaption effect that had already been established could be broken as the exposure duration continued to increase, and a new sickness adaption process would establish. Moreover, we showed a distinguishable symptom profile of HMD compared with LCD, which was insusceptible of exposure duration. This is the first report presenting the temporal pattern of VR sickness during such long-duration exposure. Our study could offer a predictive model of VR sickness severity level during long virtual immersion and provide suggestions for the use of VR technology for scientific study, clinical application, and business entertainment.
\end{abstract}

Keywords Virtual reality sickness · Virtual immersion · Sickness adaption effect · Sensory conflict $\cdot$ Long-duration exposure

\section{Introduction}

Virtual reality technology (VR) is a valuable tool that is increasingly used in neuroscientific research and clinic rehabilitation (Bohil et al. 2011; Parsons 2015; Farook et al. 2018, Szpak et al. 2020). Nevertheless, it brings us not only advantages but also health and safety side effects, such as VR sickness. VR sickness occurs when people exposed to a virtual environment (Cobb et al. 1999; Sharples et al. 2008; Kim et al. 2018). It is a bodily discomfort associated with a series of symptoms such as disorientation, nausea, vomiting, and visual fatigue. Such discomfort could induce aversive or disgusting experiences for users, and have adverse impacts on task performance and clinical rehabilitation (Stanney et al. 2002; Kim et al. 2005; Kiryu and So 2007; Nalivaiko et al. 2015; Nesbitt et al. 2017;

Dongdong Weng

crgj@bit.edu.cn

1 Beijing Engineering Research Center of Mixed Reality and Advanced Display, School of Optics and Photonics, Beijing Institute of Technology, No.5 Zhongguancun South Street, Haidian District, Beijing 100081, China

2 AICFVE of Beijing Film Academy, Haidian District, 8 Xitucheng Road, Beijing 100088, China
Szpak et al. 2019). The causes of VR sickness are not fully identified, but many factors have been found to contribute to VR sickness, including exposure duration (Stanney et al. 2002; Dużmańska et al. 2018), technological issues (e.g., time lag, the field of view) (Fernandes and Feiner 2016), display content (Mazloumi Gavgani et al. 2017, Guna et al. 2019), user's gender (Munafo et al. 2017) and user's age (Arns and Cerney 2005).

Among these factors contributing to VR sickness, exposure duration has a strong effect on the occurrence of VR sickness. Previous studies have observed that the severity of VR sickness was monotonically increasing with exposure duration (Moss et al. 2008; Moss and Muth 2011; Liu 2014). For example, Moss et al (2008), Moss and Muth (2011) measured VR sickness level every 2 min in an exposure duration of $10 \mathrm{~min}$ and found a significant positive correlation between sickness level and exposure duration. Liu (2014) found that the participants experienced more severe sickness as the exposure duration increased from 5 to $15 \mathrm{~min}$. Moreover, prolonged exposure time had a predominant influence on sickness severity, compared with other factors (So et al. 2001; Moss and Muth 2011). Although the above-mentioned studies showed the increasing tendency of VR sickness with time, the exposure durations measured in these studies were short (from 5 to $30 \mathrm{~min}$ ), thus 
the generalization of the temporal pattern of VR sickness in these studies is limited.

Evaluation of the effect of long-duration exposure on VR sickness is necessary to identify safe exposure, particularly when applying VR technology in clinical use (Saredakis et al. 2020). The aim of the current study is to explore the relationship between long-duration exposure and VR sickness. To achieve this end, we first developed a VR apparatus in which a virtual office work environment was established by imitating the real work scene. The virtual office environment could satisfy human basic needs such as daily office work, entertainment, communicating, and drinking (Guo et al. 2019). All participants were immersed in this virtual office environment for around $8 \mathrm{~h}$. The level of VR sickness was evaluated on average around every $1.5 \mathrm{~h}$, with five times of evaluation in total, using the Simulator Sickness Questionnaire (SSQ). This is, to our knowledge, the first research on the temporal pattern of VR sickness related to such long-duration virtual immersion.

\section{Materials and method}

\subsection{Experimental design}

To reduce individual sickness susceptibility, the current study was a within-subject design. Immersing the participants in a virtual office environment was compared to the control of them in a desktop computer display environment (Fig. 1a and b). That is, each participant completed two experimental sessions via two display interfaces: headmounted display (HMD) (using HTC VIVE Pro, field angle, $110^{\circ}$, resolution, $1440 * 1600$ per eye) and light crystal diode (LCD) computer display (using a 24-inch LCD computer screen, DELL Inc., USA). The two experimental sessions were scheduled at least one week apart to minimize possible adaptation effects. The participants were balanced for gender and the sequence of the two sessions was counterbalanced across all subjects.

\subsection{Participants}

Twenty-two participants (12 females, 20-27 years old) were recruited in this study. All participants were right-handed, had normal or corrected to the normal version, and had no history of mental or neurological disorders or sensory disorders. Participants were excluded if they had the experience of seasickness, car sickness, or sickness caused by seeing 3D movies. The participants were required to have a full-night sleep ( $\geq 7 \mathrm{~h}$ ) for one week before the experiment, and refrain from alcohol and caffeine consumption one day before the experiment and on the day of the experiment. Written informed consent was obtained from each participant, with

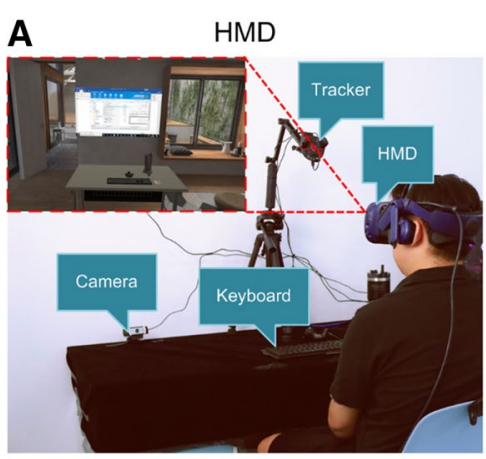

B $\quad L C D$

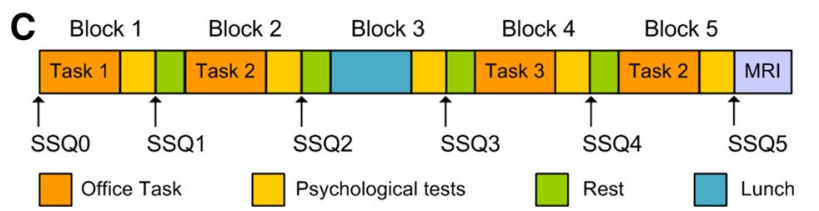

Fig. 1 The schematic diagram of the experimental protocol. Participants were required to complete four simple office tasks and several psychological tests including SSQ via HMD (a) or LCD computer display (b)

the experimental purpose and task informed and the risk of VR sickness explained. All participants were informed of their right to withdraw from the study at any time.

\subsection{Experimental protocol}

The entire experiment was conducted in a hospital. All participants came to the hospital on the day before the experiment to be familiar with the HMD and the VR environment. The experiment started at 9:00 am. All participants came to the hospital around 8:00 am to complete several pre-experimental questionnaires including the Simulator Sickness Questionnaire.

We imitated the real work scene during each experimental session in which the participants were required to perform four simple office tasks via keyboard and mouse (Fig. 1a and $b$ ). These office tasks were arranged in order, including finding wrong-written words, searching for specified content from a long article, making PowerPoint slides with a specific aim, and classifying images. The participants completed 4, 90-min blocks with 5-10 min break in between (Fig. 1c). Each block included one office task except block 3. The office task was set to last $40 \mathrm{~min}$ and stopped automatically at the end of the time. For block 3, the participants had a break of $40 \mathrm{~min}$ from 12:00 to 12:40 for lunch instead. In each block, after the office task or lunch finished, the participants were required to complete several psychological tasks, then the sickness level was evaluated using the Simulator Sickness Questionnaire. After the sickness level evaluation, the participants could take a rest (go to the toilet or listen to music) for 5-10 min. It should be noted that for the HMD 
condition, the participants kept wearing the HMD during the whole session except lunchtime and going to the toilet. The participants underwent the MRI scan immediately after they finished the sickness level evaluation in the last block.

\subsection{Simulator sickness questionnaire}

The simulator sickness questionnaire (SSQ) is a widely used scale for quantifying simulator sickness levels under VR or simulator environments (Balk et al. 2017). The SSQ comprises 16 sickness symptoms, with variable scores $0,1,2$, and 3, corresponding to 4 different degrees of severity: none, slight, moderate, and severe. The total severity (TS) of sickness symptoms is acquired by a weighted scoring procedure to obtain a total score that reflects the overall sickness level (k, 1993). The SSQ can be split into 3 distinguishable but correlated subscales including disorientation, oculomotor, and nausea, and the TS score is a composite of the three subscales.

\subsection{SSQ score analysis}

To compare the SSQ score between the HMD and the control condition (LCD), we first perform a 2 (display condition) $\times 6$ (exposure duration) repeated measures ANOVA to examine the main effect of display condition on the SSQ score. Specifically, the display condition included the HMD and LCD conditions, the exposure duration included preexperiment (T0), $1.5 \mathrm{~h}$ (T1), $3 \mathrm{~h}$ (T2), $4.5 \mathrm{~h}$ (T3), $6 \mathrm{~h}$ (T4), and $7.5 \mathrm{~h}$ (T5). Then a 1 (participant) $\times 6$ (exposure duration) repeated measures ANOVA was conducted to reveal the effect of the exposure duration on VR sickness. Posthoc pairwise Bonferroni tests were applied to SSQ score if the ANOVA had revealed a main effect. A linear regression analysis was conducted to reveal the relation between SSQ score and exposure duration. In this study, we mainly used the SSQ TS score in the analysis because it was considered the most reliable index for total sickness symptom severity (Kennedy et al. 2000).

\section{Results}

\subsection{SSQ score analysis}

SSQ scores evaluated before and during 7.5-h exposure in HMD and LCD conditions were shown in Table 1.

For SSQ TS score, as shown in Fig. 2a, the two-way repeated measures ANOVA revealed a statistically significant main effect of the display interface $\left(F_{1,21}=63.263\right.$, $p<0.0001$ ), with a significantly higher SSQ total score for HMD compared with the control condition. A significant main effect of exposure duration was also found $\left(F_{5,105}=13.608, p<0.0001\right)$. The interaction effect between the display interface and exposure duration was not significant $\left(F_{5,105}=2.787, p=0.066\right)$.

A repeated measures ANOVA revealed a significant effect of exposure duration on SSQ TS score $\left(F_{5,105}=8.885, p<0.0001\right)$ for the HMD condition. SSQ TS score significantly increased exposure time. Post-hoc Bonferroni tests showed significant differences between different exposure durations. All SSQ TS scores were significantly higher during VR exposure (T1-T5) as compared to pre-experiment (T0) (for all, $p<0.05$ ). Furthermore, the SSQ TS score was significantly increased in T4 and T5 compared with T1 (for all, $p<0.05$ ).

The variation curve of sickness symptom severity along with time was drawn for disorientation, oculomotor, and nausea subscales, respectively to reveal the relation between exposure duration and SSQ subscale (Fig. 2b-d).

Moreover, the sickness symptom profiles were distinguishable between HMD and LCD conditions. As shown in Fig. 3, the HMD showed a prominent symptom of disorientation compared with oculomotor disturbance and nausea (Fig. 3a), while the LCD showed proportionately more oculomotor disturbance than disorientation and nausea (Fig. 3b).

Table 1 SSQ analysis results

\begin{tabular}{|c|c|c|c|c|c|c|c|}
\hline \multirow[t]{2}{*}{ Interface } & \multirow[t]{2}{*}{ SSQ } & \multicolumn{6}{|c|}{ Exposure duration (Mean \pm SEM) } \\
\hline & & Pre-exposure (T0) & $1.5 \mathrm{~h}(\mathrm{~T} 1)$ & $3 \mathrm{~h}(\mathrm{~T} 2)$ & $4.5 \mathrm{~h}(\mathrm{~T} 3)$ & $6 \mathrm{~h}(\mathrm{~T} 4)$ & $7.5 \mathrm{~h}(\mathrm{~T} 5)$ \\
\hline \multirow[t]{4}{*}{ HMD } & Disorientation & $20.2 \pm 5.8$ & $44.3 \pm 7.4$ & $58.8 \pm 10.9$ & $58.5 \pm 11.3$ & $66.4 \pm 11.4$ & $74.6 \pm 12.9$ \\
\hline & Oculomotor & $12.1 \pm 2.7$ & $33.4 \pm 4.5$ & $44.4 \pm 5.4$ & $47.5 \pm 7.1$ & $55.1 \pm 6.2$ & $57.2 \pm 7.5$ \\
\hline & Nausea & $3.5 \pm 1.3$ & $12.1 \pm 2.9$ & $19.9 \pm 4.6$ & $20.5 \pm 5.0$ & $22.1 \pm 4.6$ & $25.2 \pm 5.6$ \\
\hline & Total score & $7.4 \pm 2$ & $17.6 \pm 2.7$ & $23.5 \pm 3.9$ & $24.3 \pm 4.4$ & $27.4 \pm 4.3$ & $30.2 \pm 4.9$ \\
\hline \multirow[t]{4}{*}{ LCD } & Disorientation & $7.6 \pm 4.1$ & $7.6 \pm 2.5$ & $15.8 \pm 5.4$ & $22.1 \pm 6.3$ & $25.3 \pm 6.3$ & $29.7 \pm 8.2$ \\
\hline & Oculomotor & $7.2 \pm 4.7$ & $13.8 \pm 2.8$ & $20.3 \pm 4.5$ & $26.2 \pm 5.3$ & $29.3 \pm 4.8$ & $34.5 \pm 5.9$ \\
\hline & Nausea & $5.6 \pm 3$ & $3.0 \pm 1.2$ & $10.0 \pm 2.9$ & $9.1 \pm 2.1$ & $7.4 \pm 1.7$ & $14.3 \pm 3.2$ \\
\hline & Total score & $3.6 \pm 1.7$ & $4.2 \pm 1.1$ & $8.0 \pm 2.2$ & $10.4 \pm 2.5$ & $11.4 \pm 2.4$ & $14.0 \pm 3.2$ \\
\hline
\end{tabular}


Fig. 2 Temporal pattern of SSQ scores for HMD and LCD. a-d MEANS and SEMs of SSQ scores, a significant positive linear relationship between exposure duration and total severity score (a), disorientation (b), oculomotor (c), and nausea (d) were observed specifically in both HMD and LCD. Error bars represent \pm 1 standard error of the mean (SEM)
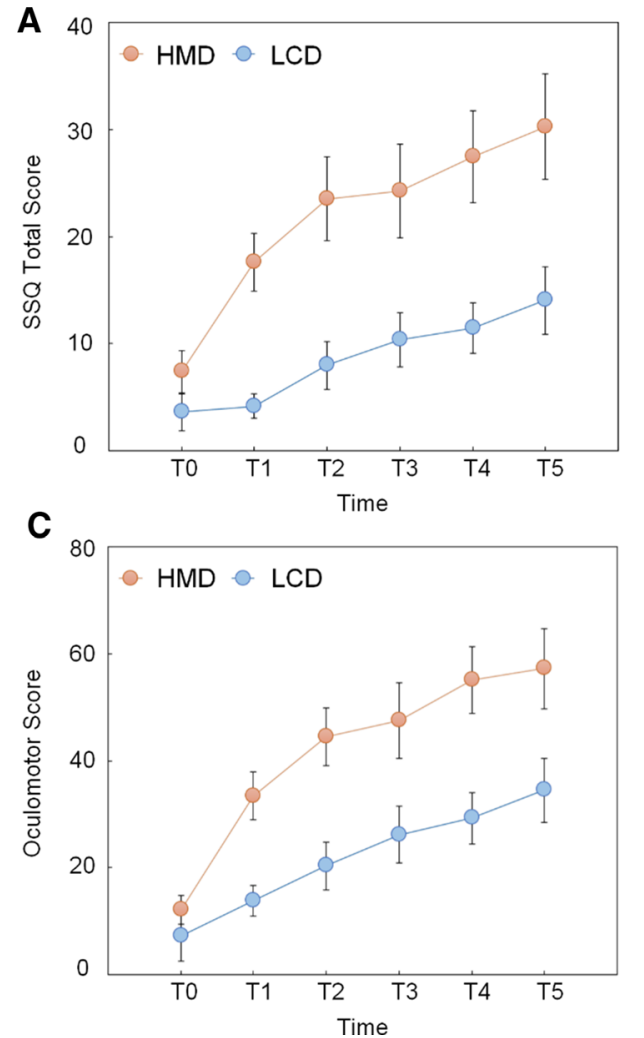
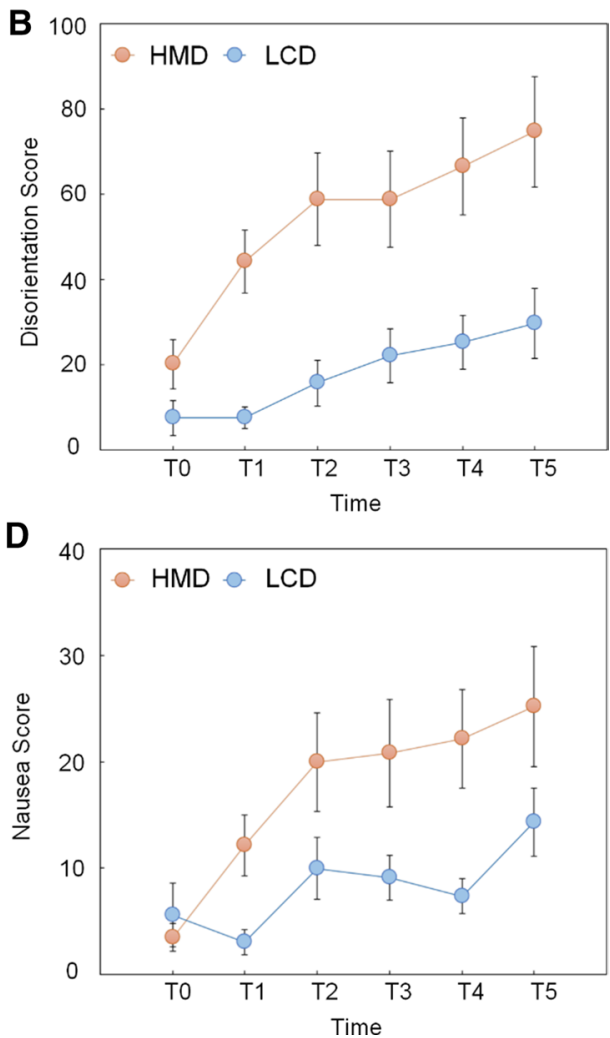

\section{A}

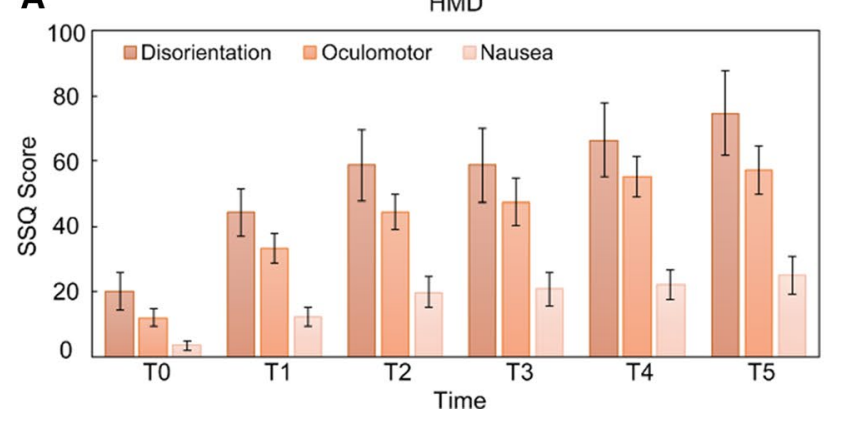

B

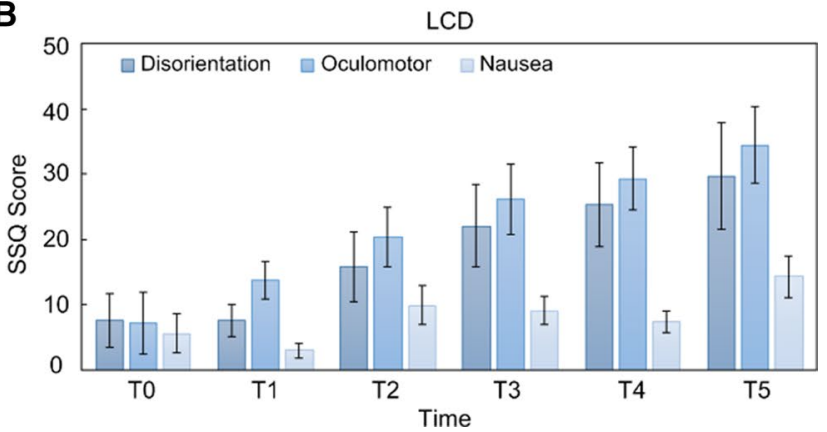

Fig. 3 Sickness symptom profiles in HMD (a) and LCD (b)

\section{Discussion}

The changing trend of VR sickness during 7.5-h virtual environment immersion has not been reported. Only one study used varying exposure duration times in different experimental data sets and to examine the influence of exposure duration on VR sickness levels during 3 or more hours of virtual exposures (Kennedy et al. 2000). However, due to the limits of the technology, the HMD devices used in the previous studies easily induced more severe sickness symptoms compared with modern HMD devices. Thus, the temporal pattern of sickness level generalized from previous experimental data sets could not be generalizable under modern experimental conditions.

Our study provided the first evidence of the temporal pattern of VR sickness during long-duration virtual immersion. Based on 7.5-h immersion in the virtual office environment, we assessed the relationship between VR sickness level and exposure duration and identified a significant positive linear relationship between sickness level and exposure duration time. Previous studies based on various approaches have found an increasing tendency of sickness levels to rise over time, despite their relatively short length of exposure time compared with our study (Stanney et al. 2002; Moss et al. 2008; Moss and Muth 2011). These results lead to 
the conclusion that the temporal pattern of VR sickness is stable despite the technological issues of the VR system (Dużmańska et al. 2018). Our results confirmed the conclusion that the VR sickness severity is more severe when the exposure duration is longer, even during such a long time virtual immersion. The temporal pattern of VR sickness identified in our study could offer a model for the prediction of the level of VR sickness based on exposure time, thus guild the design and use of VR technology for scientific study, clinical application, and business entertainment.

Previous studies showed that the severity of VR sickness could reach its peak level after a certain amount of time of immersion, which is known as the sickness adaptation effect (Lampton et al. 2000; Moss et al. 2008; Brooks et al. 2010; Sinitski et al. 2018). Our study revealed that the sickness adaption effect was not stable in the case of long-duration exposure. In the current study, total sickness severity was increased sharply as the exposure duration increased from 0 to $1.5 \mathrm{~h}$, then the increasing rate gradually became slow, with no significant difference of total sickness severity observed when compared SSQ TS scores on T1, T2, and T3. These observations implied that, during the first 4.5-h VR immersion, the participants had severe VR sickness symptoms first as they were exposed from the real world to the virtual world, then they gradually adjusted themselves to the virtual environment so that the sickness adaptation was achieved. However, as the exposure time continued to increase, the sickness adaptation was broken, which was verified by the significantly higher level of total sickness observed on T4 compared with T1. Our results indicated that after a period of time of immersion, a threshold of sickness level appeared and persisted for some time (T1-T3), then the sickness adaptation effect began to diminish and a new threshold level showed up as the time of exposure continues to increase (T4-T5), revealing a dynamic process of sickness adaptation during a long time of VR exposure.

Our study also provided the direct comparison of symptom profiles and total sickness symptom between HMD and LCD monitor among five duration categories. The symptom profiles of the two display interfaces were quite distinguishable. Disorientation was the prominent symptom for HMD, while the LCD monitor showed proportionately more symptoms of oculomotor disturbance compared with nausea and disorientation. Sharples et al. (2008) compared sickness characteristics of HMD against the desktop computer, reality theatre, and projection screen after 30-min exposure in an interactive virtual environment and found significantly higher SSQ TS and disorientation scores in comparison with desktop computer and reality theatre. More recently, Guna et al. (2019) studied the sickness characteristics of four types of VR HMDs and 2D TV screens and found similar results in both neutral and action video display conditions. The specific feature of VR sickness was also identified when compared with a flight simulator (Kennedy et al. 2003). Taken together, these studies show that VR sickness has distinguishable sickness characteristics manifested in severity level and symptom profile, compared with sickness induced by LCD monitor and simulator sickness.

Funding This study was supported by the National Key Research and Development Program of China (No.2018YFF0300802), the National Natural Science Foundation of China (No.61902026), the National Natural Science Foundation of China (No. U1605254), and the 111 Project (B18005).

\section{Declarations}

Conflict of interest The authors declare no conflict of interest.

Ethical approval All procedures performed in studies involving human participants were in accordance with the ethical standards of the institutional and/or national research committee and with the 1964 Helsinki declaration and its later amendments or comparable ethical standards.

Informed consent Informed consent was obtained from all individual participants included in the study.

Open Access This article is licensed under a Creative Commons Attribution 4.0 International License, which permits use, sharing, adaptation, distribution and reproduction in any medium or format, as long as you give appropriate credit to the original author(s) and the source, provide a link to the Creative Commons licence, and indicate if changes were made. The images or other third party material in this article are included in the article's Creative Commons licence, unless indicated otherwise in a credit line to the material. If material is not included in the article's Creative Commons licence and your intended use is not permitted by statutory regulation or exceeds the permitted use, you will need to obtain permission directly from the copyright holder. To view a copy of this licence, visit http://creativecommons.org/licenses/by/4.0/.

\section{References}

Arns LL, Cerney MM (2005) The relationship between age and incidence of cybersickness among immersive environment users. In: IEEE proceedings. VR 2005. Virtual reality, 2005.

Balk S, Bertola M, Inman V (2017) Simulator sickness questionnaire: twenty years later

Bohil CJ, Alicea B, Biocca FA (2011) Virtual reality in neuroscience research and therapy. Nat Rev Neurosci 12(12):752-762

Brooks JO, Goodenough RR, Crisler MC, Klein ND, Alley RL, Koon BL, Logan WC Jr, Ogle JH, Tyrrell RA, Wills RF (2010) Simulator sickness during driving simulation studies. Accid Anal Prev 42(3):788-796

Cobb SVG, Nichols S, Ramsey A, Wilson JR (1999) Virtual realityinduced symptoms and effects (VRISE). Presence 8(2):169-186

Dużmańska N, Strojny P, Strojny A (2018) Can simulator sickness be avoided? A review on temporal aspects of simulator sickness. Front Psychol 9:2132-2132

Farook S, Singh K, Govind S, Kesvan R, Chinnavan E (2018) Impact of virtual reality training in rehabilitation of stroke patients: a cross sectional review 8 
Fernandes AS, Feiner SK (2016) Combating VR sickness through subtle dynamic field-of-view modification. In: 2016 IEEE symposium on 3D user interfaces (3DUI).

Guna J, Geršak G, Humar I, Orel M, Krebl M, Lu H, Pogacnik M (2019) Virtual reality sickness and challenges behind different technology and content settings. Mobile Networks Appl

Guo J, Weng D, Zhang Z, Liu Y, Wang Y (2019) Evaluation of maslows hierarchy of needs on long-term use of HMDs-a case study of office environment. In: 2019 IEEE conference on virtual reality and 3D user interfaces (VR)

Kennedy RS, Stanney K, Dunlap WP (2000) Duration and exposure to virtual environments: sickness curves during and across sessions. Presence Teleoperat Virt Environ 9:463-472

Kennedy RS, Drexler JM, Compton DE, Stanney KM, Lanham DS, Harm DL (2003) Configural scoring of simulator sickness, cybersickness, and space adaptation syndrome: Similarities and differences. Virtual and adaptive environments: applications, implications, and human performance issues. Mahwah, NJ, US: Lawrence Erlbaum Associates Publishers, 247-278

Kim YY, Kim HJ, Kim EN, Ko HD, Kim HT (2005) Characteristic changes in the physiological components of cybersickness. Psychophysiology 42(5):616-625

Kim HK, Park J, Choi Y, Choe M (2018) Virtual reality sickness questionnaire (VRSQ): motion sickness measurement index in a virtual reality environment. Appl Ergon 69:66-73

Kiryu T, So RHY (2007) Sensation of presence and cybersickness in applications of virtual reality for advanced rehabilitation. J Neuroeng Rehabil 4(1):34

Lampton DR, Rodriguez ME, Cotton JE (2000) Simulator sickness symptoms during team training in immersive virtual environments. Proceed Human Factors Ergonom Soc Ann Meet 44(5):530-533

Liu C-L (2014) A study of detecting and combating cybersickness with fuzzy control for the elderly within 3D virtual stores. Int J Hum Comput Stud 72(12):796-804

Mazloumi Gavgani A, Hodgson DM, Nalivaiko E (2017) Effects of visual flow direction on signs and symptoms of cybersickness. PLOS One 12(8):e0182790

Moss JD, Muth ER (2011) Characteristics of head-mounted displays and their effects on simulator sickness. Hum Factors 53(3):308-319

Moss J, Scisco J, Muth E (2008) Simulator sickness during head mounted display (HMD) of real world video captured scenes. Proceed Human Factors Ergonom Soc Ann Meet 52:1631-1634
Munafo J, Diedrick M, Stoffregen T (2017) The virtual reality headmounted display Oculus Rift induces motion sickness and is sexist in its effects. Exp Brain Res 235

Nalivaiko E, Davis SL, Blackmore KL, Vakulin A, Nesbitt KV (2015) Cybersickness provoked by head-mounted display affects cutaneous vascular tone, heart rate and reaction time. Physiol Behav 151:583-590

Nesbitt K, Davis S, Blackmore K, Nalivaiko E (2017) Correlating reaction time and nausea measures with traditional measures of cybersickness. Displays 48:1-8

Parsons TD (2015) Virtual reality for enhanced ecological validity and experimental control in the clinical, affective and social neurosciences. Front Hum Neurosci 9:660-660

Saredakis D, Szpak A, Birckhead B, Keage HAD, Rizzo A, Loetscher T (2020) Factors associated with virtual reality sickness in headmounted displays: a systematic review and meta-analysis. Front Human Neurosci 14(96)

Sharples S, Cobb S, Moody A, Wilson JR (2008) Virtual reality induced symptoms and effects (VRISE): comparison of head mounted display (HMD), desktop and projection display systems. Displays 29(2):58-69

Sinitski EH, Thompson AA, Godsell P, Honey J, Besemann M (2018) Postural stability and simulator sickness after walking on a treadmill in a virtual environment with a curved display. Displays $52: 1-7$

So RH, Lo WT, Ho AT (2001) Effects of navigation speed on motion sickness caused by an immersive virtual environment. Hum Factors 43(3):452-461

Stanney KM, Kingdon KS, Graeber D, Kennedy RS (2002) Human performance in immersive virtual environments: effects of exposure duration, user control, and scene complexity. Hum Perform 15(4):339-366

Szpak A, Michalski SC, Saredakis D, Chen CS, Loetscher T (2019) Beyond feeling sick: the visual and cognitive aftereffects of virtual reality. IEEE Access 7:130883-130892

Publisher's Note Springer Nature remains neutral with regard to jurisdictional claims in published maps and institutional affiliations. 